\title{
A new approach for classification of human brain CT images based on morphological operations
}

\author{
Ali Reza Fallahi ${ }^{1}$, Mohammad Pooyan ${ }^{1}$, Hassan Khotanlou ${ }^{2}$ \\ ${ }^{1}$ Biomedical Engineering Department, Shahed University, Tehran, Iran; \\ ${ }^{2}$ Computer Engineering Department, Bu-Ali Sina University, Hamedan, Iran. \\ Email: afallahi@shahed.ac.ir; pooyan@shahed.ac.ir; hkh@basu.ac.ir
}

Received 5 September 2009; revised 30 September 2009; accepted 8 October 2009.

\begin{abstract}
Automatic diagnosis may help to decrease human based diagnosis error and assist physicians to focus on the correct disease and its treatment and to avoid wasting time on diagnosis. In this paper computer aided diagnosis is applied to the brain CT image processing. We compared performance of morphological operations in extracting three types of features, i.e. gray scale, symmetry and texture. Some classifiers were applied to classify normal and abnormal brain CT images. It showed that morphological operations can improve the result of accuracy. Moreover SVM classifier showed better result than other classifiers.
\end{abstract}

Keywords: CT Image; Feature Extraction; Classification; Morphological Operations; Automatic Diagnosis

\section{INTRODUCTION}

Medical CT image has been applied in clinical diagnosis widely. It can assist physicians to detect and locate pathological changes, and determine the property of them. But the diagnosis result is often subjective, different physicians may get different diagnosis result at different time [1]. Computer Aided Diagnosis (CAD) aims to provide a computer output as a second opinion in order to assist physicians in the detection of abnormalities, quantification of disease progress and differential diagnosis of lesions [2]

The typical architecture of a CAD system (Figure 1) includes four main modules: image pre-processing, definition of region(s) of interest (ROI), extraction and selection of features and classification of the selected ROI [3].

The aim of image pre-processing is to improve the quality of data through the application of methods for denoising such as mean, median, Laplacian and Gaussian filters and enhancing the edges of image structures such as unsharpening and wavelet transform and enhancing image contrast like histogram equalization.
Feature extraction refers to various quantitative measurements of medical images typically used for decision making related to the pathology of a structure or tissue. When the features have been extracted, selection of a subset of the most robust features is essential, aiming at improving classification accuracy and reducing the overall complexity. Some methods such as PCA, LDA and genetic algorithms can be used for this purpose.

One of the major problems of pattern recognition in medical image analysis is the classification of a set of features into the proper classes. The main methods in the brain CT images classification include RBFNN [1], decision tree, See5, inductive learning [4] and Bayesian.

In this paper we applied morphological operations to the images and then extracted three types of features. We then evaluated performance of these operations and extracted features by six types of classifiers.

\section{MATERIALS AND METHOD}

There are lots of diseases that originate in the brain. The CT image is the lamination cross-sectional image. Different stratification plane images have different patterns. In order to get the better image analysis, we select one kind of disease to determine whether it is normal or abnormal and to extract features from one plane in the CT of brain image (Figure 2). The ordinary utilized features are texture, gray scale, shape and symmetry. Our experiment focuses on texture, symmetry, gray scale and their formulation.

\subsection{Gray Scale Feature}

In digital image processing, the two-dimensional digitized gray scale image $(M \times N)$ can be seen as $M \times N$ pixels in two-dimensional surface XOY, each pixel (x,y) can be represented as its gray value. Grayscale features that can be extracted are mean, variance, skewness and kurtosis. Among them, the standard variance reflects the separate degree of gray scale value. The skewness takes the mean value as the central data distribution. Skewness is represented by the following equation: 


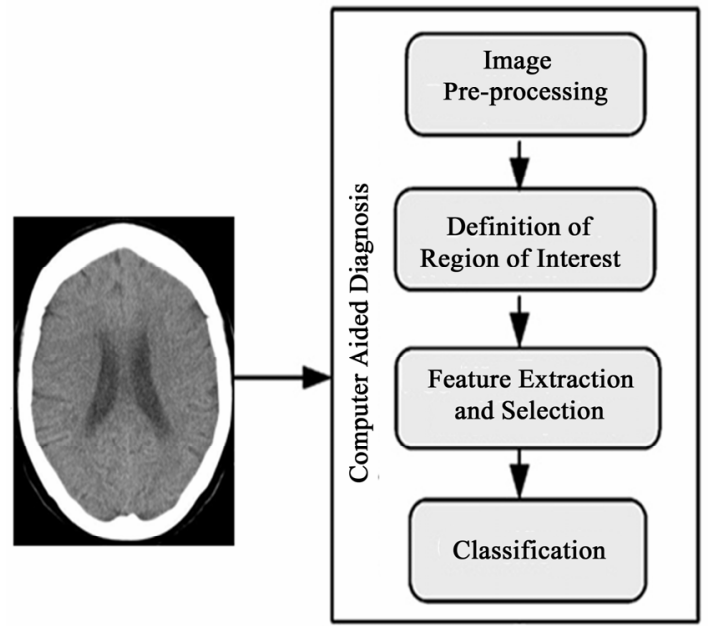

Figure 1. Architecture of a CAD system.

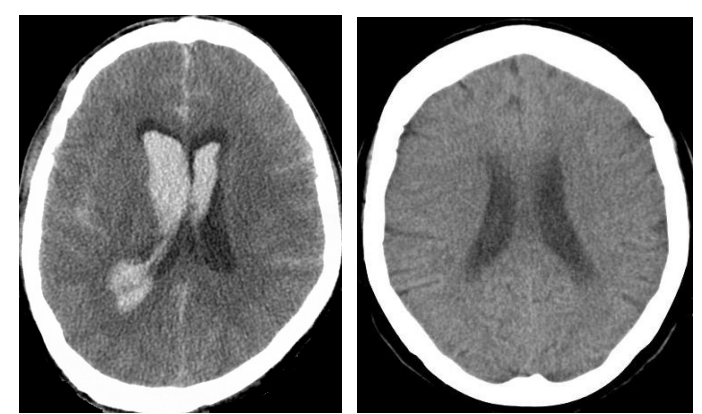

Figure 2. Abnormal and normal brain CT images.

$$
M_{c 3}=\left(\frac{1}{M_{c 2}{ }^{3}}\right) \sum_{x=1}^{m} \sum_{y=1}^{n}\left(f(x, y)-M_{c 1}\right)^{3}
$$

The kurtosis reflects the normal distribution sharpness or smoothness of the compared data that is represented by the following equation:

$$
M_{c 4}=\left(\frac{1}{M_{c 2}{ }^{4}}\right) \sum_{x=1}^{m} \sum_{y=1}^{n}\left(f(x, y)-M_{c 1}\right)^{4}
$$

Because of symmetry structure of the brain, we divide image in two partitions. The above features are extracted in the left and right sections. We then calculate the Euclidian distance corresponding to four features of two sections as the new features. We applied these new features for classification.

\subsection{Texture Features}

A co-occurrence matrix (COM) is the square matrices of relative frequencies $P(i, j, d, \theta)$ with which two neighboring pixels separated by the distance $d$ at the orientation $\theta$ occur in the image (Figure 3), one with gray level $\mathrm{i}$ and the other with gray level $\mathrm{j}$ [5]. A COM is therefore a square matrix that has the size of the largest pixel value in the image and present the relative frequency distribu-

tions of gray levels and describe how often one gray level will appear in a specified spatial relationship to another gray level within each image region [6]. There are 14 features that may be extracted from COM matrix, but usually four or five features are more interested ones. In this paper four textural features were calculated from the COM for direction $h$ values of $0^{\circ}$ and a distance $d$ of 1 .

The matrix was normalized by the following function:

$$
p(i, j, d, \theta)=\frac{p(i, j, d, \theta)}{R}
$$

$\mathrm{R}$ is the normalized function, which is usually set as the sum of the matrix.

Energy is also called Angular Second Moment. It is a measure of the homogeneousness of the image and can be calculated from the normalized COM. It is a suitable measure to detect disorder in texture image. Higher values for this feature mean less changes in the image amplitude or intensity result in a much sparser COM.

The energy is formulated by the following equation:

$$
J=\sum_{i=1} \sum_{j=1}(p(i, j))^{2}
$$

Contrast is a measure of amount of the local variation in the image. It will have a large value for images which have a large amount of local variation in gray levels and a smaller value for images with uniform gray level distributions and is defined as:

$$
G=\sum_{i=1} \sum_{j=1}(i-j)^{2} p(i, j)
$$

The Inverse Difference Moment (IDM) reflects the local texture changes. It is another feature of image contrast and is defined as:

$$
I D M=\sum_{i=1} \sum_{j=1} \frac{1}{1+(i-j)^{2}} p(i, j)
$$

Entropy gives a measure of complexity of the image. Complex textures tend to have higher entropy. Entropy is represented by the following equation:

$$
S=-\sum_{i=1} \sum_{j=1} p(i, j) \log (p(i, j))
$$

\section{0 degrees}

135 degress

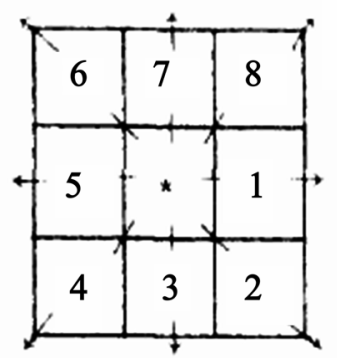

Figure 3. Co-accurance matrix calculation.
45 degrees

0 degrees 

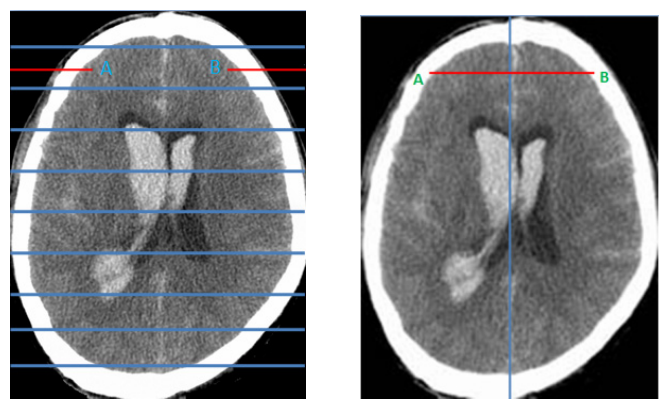

Figure 4. Exterial symmetry (left) and interial symmetry (right) feature calculation.
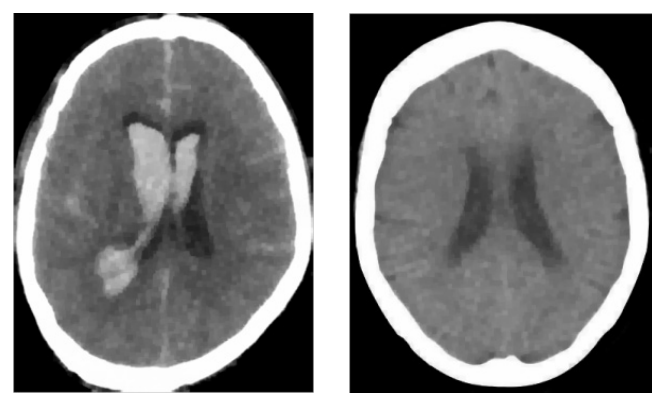

Figure 5. Result of applying morphological operation.

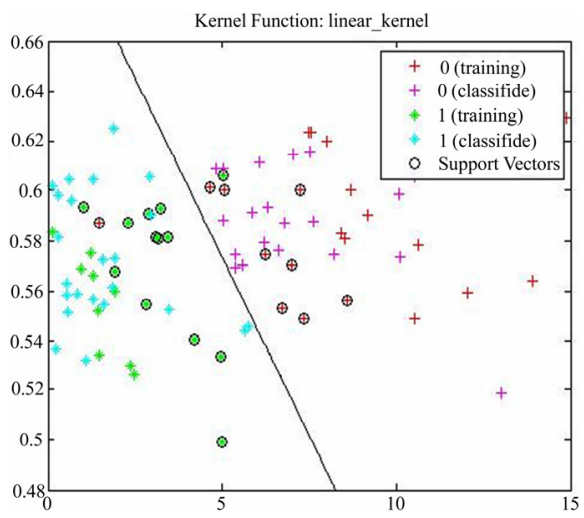

Figure 6. Result of classifing data with SVM.

\subsection{Symmetrical Feature}

Symmetrical features including two types: Interior symmetry and exterior symmetry.

1) Interior symmetry: We partition one image in ten parts with same length, where $p$ is the part number, $S_{i}$ is the sum of symmetry pixel points in each part, $V_{i}$ is the sum of all the pixel points in each part. If $x-y \leq 4$ then point A is symmetrical to point B. $x$ is the gray scale value of point $\mathrm{A}$ and $y$ is the gray scale value of point $B$. From the two edges of each part to the center, for detecting the interior symmetrical pixel point their sum per pixel line is calculated. In a symmetrical images, the value of $S y_{p}$ is greater than an unsymmet- rical images. Therfore, the value of $S y_{p}$ in the normal images is greater than the abnormal ones. Interior symmetry is defined as:

$$
S y_{p}=\sum_{i=1}^{n} s_{i}
$$

2) Exterior symmetry: Let us $m_{i}$ represents the $X$ coordinate value of midpoint of each pixel line in image, $n$, the height of the image and $M$ the average $\mathrm{X}$ coordinate value of all the pixel line in image (Figure 4). The more symmetrical image has higher value of $S_{E}$. But, if there are pathological changes in the image, the value of $S_{E}$ is uncertain. Exterior symmetry is defined as:

$$
S_{E}=\frac{\sum_{i=1}^{n}\left(m_{i}-M\right)^{2}}{n}
$$

\subsection{Morphological Operations}

Morphological operations are capable of deleting small disconnected regions, filling cavities and smoothing the region-of-interest [7]. Tow types of digital morphological operations, opening followed by closing, are applied to the image to eliminate the small isolated regions. These operations are defined as ordered combinations of fundamental operations, dilation and erosion. If the translation operation is defined as

$$
\mathbf{A}+x=\{a+x: a \in \mathbf{A}\}
$$

Then the operators' erosion and dilation can be written as:

$$
\begin{gathered}
E(\mathrm{~A}, \mathrm{~B})=\mathrm{A} \Theta \mathrm{B}=\{x:-\mathrm{B}+x \subset \mathrm{A}\} \\
D(\mathbf{A}, \mathbf{B})=\mathbf{A} \oplus \mathbf{B}=\{x:(-\mathbf{B}+x) \cap \mathbf{A}=\phi\}
\end{gathered}
$$

where $\mathrm{A}$ is the image and $\mathrm{B}$ is the structuring element. Note that $-\mathrm{B}$ denotes the reflection of $\mathrm{B}$ with regard to the origin, $\mathrm{x}$ denotes a point in space, and $a$ is a point in the image A [8]. Here we use circular element with radius $r$ which can be determined by a compromise between the noise suppression performance and preservation of details.

\subsection{Classification}

We selected a set of 6 well known classifiers that their computation procedures would be described briefly in the following sections.

1) Linear Bayes Normal Classifier: This computes the linear classifier between the classes of the dataset by assuming normal densities with equal covariance matrices. The joint covariance matrix is the weighted by a priori probabilities average of the class covariance ma- 
Table 1. Accuracy of classifiers without morphological operations.

\begin{tabular}{|c|c|c|c|c|}
\hline Classes & Symmetry & Grayscale & Texture & total \\
\hline 1 : Bayes-Normal-(Quadratic) & 97.75 & 66.75 & 59 & 98.5 \\
\hline 2 : Bayes-Normal-(linear) & 99.25 & 67.75 & 67.5 & 99.25 \\
\hline 3: K-NN Classifier & 98.5 & 66.75 & 37.25 & 45.2 \\
\hline 4: Decision Tree & 89.25 & 54 & 62.75 & 83 \\
\hline 5.PP 5 hidden neuron & 93 & 74 & 79 & 95 \\
\hline 10 hidden neuron & 97 & 73 & 67 & 90 \\
\hline 6:SVM & 97 & 88 & 90 & 100 \\
\hline
\end{tabular}

Table 2. Accuracy of classifiers with morphological operations.

\begin{tabular}{|c|c|c|c|c|}
\hline Classes & Symmetry & Grayscale & Texture & total \\
\hline 1 : Bayes-Normal-(Quadratic) & 98.5 & 58.25 & 61.25 & 100 \\
\hline 2 : Bayes-Normal-(linear) & 99.25 & 70 & 64 & 100 \\
\hline 3: K-NN Classifier & 100 & 65.75 & 45.25 & 45 \\
\hline 4: Decision Tree & 91.25 & 45.75 & 54 & 88.75 \\
\hline 5 hidden neuron & 100 & 85 & 75 & 97 \\
\hline 10 hidden neuron & 96 & 81 & 65 & 95 \\
\hline $6: S V M$ & 97 & 90 & 86 & 100 \\
\hline
\end{tabular}

Table 3. Result of other papers.

\begin{tabular}{cccccc}
\hline Author & classifier & Symmetry & Grayscale & Texture & Total \\
\hline \multirow{2}{*}{ Zhang et.al } & See5 & $85-95$ & $72-76$ & $75-78$ & $90-94$ \\
& RBF NN & $85-89$ & $72-75$ & $62-65$ & $83-86$ \\
& See5 & $85-93$ & - & $75-78$ & - \\
\multirow{2}{*}{ Wang et.al } & RBF NN & $85-89$ & - & $67-71$ & - \\
& Outlier & $91-98.5$ & - & $76-80$ & - \\
& Detection & & & \\
\hline
\end{tabular}

trices. The covariance matrix of the classes is then decomposed as $\mathrm{G}=\mathrm{W}^{*} \mathrm{~W}^{\prime}+\operatorname{sigma}^{\wedge} 2 *$ eye $(\mathrm{K})$, where $\mathrm{W}$ is a $\mathrm{K} \times \mathrm{M}$ matrix containing the $\mathrm{M}$ leading principal components and sigma ${ }^{\wedge} 2$ is the mean of the K-M smallest eigenvalues. Finally, the classification is computed.

2) Quadratic Bayes Normal Classifier: It also computes the quadratic classifier between the classes of the dataset, assuming normal densities. The covariance matrix of the classes is then decomposed as $\mathrm{G}=\mathrm{W}^{*} \mathrm{~W}^{\prime}+$ sigma $^{\wedge} 2 *$ eye $(\mathrm{K})$, where $\mathrm{W}$ is a $\mathrm{K} \times \mathrm{M}$ matrix, containing the $\mathrm{M}$ leading principal components and sigma ${ }^{\wedge}$ is the mean of the K-M smallest eigenvalues. The classification is then performed.

3) K-NN Classifier: It computes the common Knearest neighbor classifier for the dataset.

4) Decision Tree: It computes a decision tree classifier out of a dataset using a binary splitting criterion.

5) Back Propagation Neural Classifier: A feedforward neural network classifier with five and ten hidden units in the second layer that number of input neurons depends on number of features and hidden neurons are selected manually is computed for the dataset.

6) SVM (Support Vector Machines): SVM is a new classification method for both linear and nonlinear data. It uses a nonlinear mapping to transform the original training data into a higher dimension. With the new dimension, it searches for the linear optimal separating hyperplane. SVM finds this hyperplane using support vectors ("essential" training tuples) and margins that defined by the support vectors (Figure 5). With an appropriate nonlinear mapping to a sufficiently high dimension, data from two classes can always be separated by a hyperplane.

\section{RESULTS}

To evaluate the method, we used 88 images, which 44 images are normal and the remaining is abnormal. We selected $70 \%$ of dataset as training data and $30 \%$ as testing data. Table 1 and 2 shows the result of classifiers for two cases: features with morphological operation and features without them. We extracted symmetry, gray scale and texture features with dimension of 10,4 and 5 respectively. We used a toolbox written for MATLAB named PRTools. This toolbox consists of a complete and useful set of functions for pattern recognition and includes most of the well known classification algorithms [11].

Most of the classifiers showed that the morphological operations improve results in using symmetry and gray scale features and all of them showed reduction results in texture feature that can be caused by reduction of statistical variation in the image because of applying morphological operations. Table 1 and 2 show that the result of SVM is more accurate than the other classifiers. When a mixture of the features is used, results are better than using the features sole except K-NN and Decision 
Tree, that may caused by increasing complexity. Using 5 neurons to run BPNN led better results than using 10 neurons for it. It should be noted that using 10 neurons may result in overfitting problem that reduces accuracy of the test. Table 3 shows the result of another works in this area and we can see that our method, using SVM and morphological operation can improve these results.

\section{CONCLUSIONS}

In this study, we applied morphological operations to the images and then extracted the features. Analysis result from classifiers shows that these operations can improve classification result in symmetry and grayscale features but reduce results in texture features. According to Table 1 and 2, we conclude that by using SVM classifier, we can classify the patients more accurately into the corresponding groups. Because of the brain symmetrical structure, symmetrical features have best accuracy. Our experiments show that texture features have lower accuracy. In the future works we can apply another feature extraction and feature selection techniques to improve classification accuracy.

\section{REFERENCES}

[1] Zhang, W.L. and Wang, X.Z. (2007) Extraction and classification for human brain CT images. Proceedings of Sixth International Conference on Machine learning and cybernetics, IEEE., 1155-1156.

[2] Stoitsis, J., Valavanis, I., Valavanis, S.G., Golemati, S., Nikita, A. and Nikita K.S. (2006) Computer aided diagnosis based on medical image processing and artificial intelligence methods, Nuclear Instruments and Methods in Physics Research, 569, 591-595.

[3] Haruka, D. and Teruak, A. (2007) Characterization of spatiotemporal stress distribution during food fracture by image texture analysis methods. Journal of Food Engineering, 81, 429-436.

[4] Wang, X.Z. and Lin, W.X. (2007) Application of inductive learning in human brain CT image recognition. Proceedings of Sixth International Conference on Machine learning and cybernetics, IEEE., 1155-1156.

[5] Haralick, R.M., Shanmugam, K. and Dinstein, I. (1973) Textural features for image classification. IEEE Transactions on Systems, Man, and Cybernetics, SMC-3, 6, 610-621

[6] Haruka, D. and Teruak, A. (2007) Characterization of spatiotemporal stress distribution during food fracture by image texture analysis methods, Journal of Food Engineering, 81, 429-436.

[7] Giardina, C.R. and Dougherty, E.R. (1988) Morphological Methods in Image and Signal Processing. Englewood Cliffs, NJ, Prentice-Hal.

[8] Chen, C.W., Luo, J. and Parker, K.J. (1998) Segmentation via adaptive K-Mean clustering and knowledgebased morfological operations with biomedical applications, IEEE Trans, Image Processing, Vol. 7, 12, 16731683.

[9] Mitchell, T.M.(2003) Machin learning. China Machine Press, Beijing.

[10] Mandayam, S. and Policar, R. Artificial neural networks. Lecture Note.

[11] Duin, R.P.W., Juszczak, P., Paclik, P., Pekalska, E., de Ridder, D. and Tax, D.M.J. (2004) A matlab toolbox for pattern recognition. PRTools4, Delft University of Technology. 